\author{
Marquette University \\ e-Publications@Marquette
}

6-2015

\title{
Relating the Experimental lonization Coefficients in Semiconductors to the Nonlocal Ionization Coefficients
}

Jeng Shiuh Cheong

University of Sheffield

Majeed M. Hayat

Marquette University, majeed.hayat@marquette.edu

Xinxin Zhou

University of Sheffield

John P.R. David

University of Sheffield

Follow this and additional works at: https://epublications.marquette.edu/electric_fac

Part of the Computer Engineering Commons, and the Electrical and Computer Engineering Commons

\section{Recommended Citation}

Cheong, Jeng Shiuh; Hayat, Majeed M.; Zhou, Xinxin; and David, John P.R., "Relating the Experimental Ionization Coefficients in Semiconductors to the Nonlocal Ionization Coefficients" (2015). Electrical and Computer Engineering Faculty Research and Publications. 561.

https://epublications.marquette.edu/electric_fac/561 
Marquette University

e-Publications@Marquette

\title{
Electrical and Computer Engineering Faculty Research and Publications/College of Engineering
}

This paper is NOT THE PUBLISHED VERSION; but the author's final, peer-reviewed manuscript. The published version may be accessed by following the link in th citation below.

IEEE Transactions on Electron Devices, Vol. 62, No. 6 (June 2015): 1946-1952. DOI. This article is (C) Institute of Electrical and Electronic Engineers (IEEE) and permission has been granted for this version to appear in e-Publications@Marquette. Institute of Electrical and Electronic Engineers (IEEE) does not grant permission for this article to be further copied/distributed or hosted elsewhere without the express permission from Institute of Electrical and Electronic Engineers (IEEE).

\section{Relating the Experimental lonization Coefficients in Semiconductors to the Nonlocal Ionization Coefficients}

\author{
Jeng Shiuh Cheong \\ Department of Electronic and Electrical Engineering, University of Sheffield, Sheffield, U.K. \\ Majeed M. Hayat \\ Department of Electrical and Computer Engineering, University of New Mexico, Albuquerque, NM \\ Xinxin Zhou \\ Department of Electronic and Electrical Engineering, University of Sheffield, Sheffield, U.K. \\ John P. R. David \\ Department of Electronic and Electrical Engineering, University of Sheffield, Sheffield, U.K.
}




\section{Abstract}

The experimentally determined impact ionization coefficients, $\alpha^{\prime}\left(\beta^{\prime}\right)$, include intrinsically the presence of a dead-space, where carriers cannot impact ionize as they do not have sufficient energy. These, therefore, cannot be used by nonlocal ionization models, which require the enabled ionization coefficients, $\alpha^{*}\left(\beta^{*}\right)$, which describe the ionization probability after the dead-space. A relatively simple relationship is shown to exist between $\alpha^{\prime}\left(\beta^{\prime}\right)$ and $\alpha^{*}\left(\beta^{*}\right)$,, which requires only the knowledge of the carrier threshold energies. This allows $\alpha^{\prime}\left(\beta^{\prime}\right)$, conventionally limited to the local model framework, to be used to give a very good prediction of the avalanche multiplication and excess noise for a wide range of device widths down to $0.05 \mu \mathrm{m}$, where the dead-space effect is significant. Parameterized values of $\alpha^{\prime}\left(\beta^{\prime}\right)$ and the carrier threshold energies are listed for a range of commonly used III-V semiconductors lattice matched to GaAs and InP substrates, as well as Si and SiC.

\section{SECTION I. Introduction}

Avalanche photodiodes (APDs) are widely employed in optical systems, particularly where low-light detection is necessary due to their superior sensitivity compared with $\mathrm{p}-\mathrm{i}-\mathrm{n}$ photodiodes. While a high avalanche gain is desirable, the excess noise factor due to the stochastic nature of impact ionization eventually limits the sensitivity of APDs. It is therefore important to have a model to predict the multiplication and excess noise of these devices in order to optimize their performance.

McIntyre [1] proposed an analytical model to compute the mean multiplication of any arbitrary electric-field profile resulting from a carrier-pair injection at position $\mathrm{x}$ within a multiplication region; the formula for the mean multiplication is given by

$M(x)=\frac{\exp \left[-\int_{x}^{w}\left[\alpha\left(x^{\prime}\right)-\beta\left(x^{\prime}\right)\right] d x^{\prime}\right]}{1-\int_{0}^{w} \alpha\left(x^{\prime}\right) \exp \left[-\int_{x^{\prime}}^{w}\left[\alpha\left(x^{\prime \prime}\right)-\beta\left(x^{\prime \prime}\right) d x^{\prime \prime}\right]\right] d x^{\prime}}$

This model assumes that the position-dependent ionization coefficient for electrons $(\alpha)$ and holes $(\beta)$ is solely dependent on the local electric-field strength. This simple local model allows extraction of $\alpha(\beta)$ from multiplication measurements due to pure electron and hole injection $\left(M_{e}\right.$ and $\left.M_{h}\right)$ ) [2]. Mclntyre [1] also formulated a noise theory to determine the excess noise factor $F_{e}\left(F_{h}\right)$ associated with $\left.M_{e}\left(M_{h}\right)\right)$ as

$F=k M+(1-k)\left(2-\frac{1}{M}\right)$

where $k$ is the ionization ratio given by $\beta / \alpha$ and $\alpha / \beta$ associated with $M_{e}$ and $M_{h}$ ), respectively. The excess noise decreases as $k$ approaches 0 , with the carrier type more likely to ionize, initiating the multiplication. However, this local model is highly simplified, as it assumes that the impact ionization probability depends only on the local ionization coefficients where the carrier is, whereas, realistically, it may be necessary for a carrier to travel a certain distance in an electric field to gain sufficient energy before it can ionize, i.e., the dead-space distance, $d_{e}\left(d_{h}\right)$. Early measurements of impact ionization coefficients were undertaken on thick bulk-like structures, where the dead-spaces are small relative to the device dimensions and in which case, (1) and (2) are accurate. As device dimensions decreases, the dead-spaces result in device-dependent measured ionization coefficients, $\alpha_{\text {device }}\left(\beta_{\text {device}}\right)$, which increasingly deviate from the local values of $\alpha(\beta)$ as the avalanching width of a device reduces, particularly at low electric fields [3]-[4][5], i.e., $\alpha_{\text {device }}\left(\beta_{\text {device}}\right)$ depends strongly on the width of the high-field region. One simplistic yet convenient method for determining the multiplication (or avalanche gain) in such structures is by changing the limits to the integral in (1) [3], [4], [6] to account for the dead-space while using certain device-independent ionization coefficients, $\alpha^{\prime}\left(\beta^{\prime}\right)$, which are only functions of the electric field. The quantities $\alpha^{\prime}\left(\beta^{\prime}\right)$ are available for many semiconductor materials in the literature and have often been obtained by correcting $\alpha_{\text {device }}\left(\beta_{\text {device}}\right)$, to account for the effect of the dead-space on the 
multiplication. While multiplication characteristics obtained in this manner work quite well over a range of electric fields and device dimensions down to $0.1 \mu \mathrm{m}$ [7], [8], this technique does not allow accurate prediction of the excess noise when the dead-space effects are important [9], [10].

One of the first models to successfully predict the excess noise and multiplication in devices with thin submicrometer avalanche widths was developed by Hayat et al. [11], who numerically solved a set of two coupled recurrence equations, which incorporate the carriers' ionization probability density function (pdf) in the presence of dead-space. Their dead-space multiplication theory (DSMT) is capable of calculating the multiplication and excess noise of $p$-i-n devices down to avalanche widths of $0.05 \mu \mathrm{m}[12]$, [13].

However, the accuracy of the DSMT model critically depends on an accurate knowledge of the ionization pdf. The ionization pdf requires the knowledge of $d_{e}\left(d_{h}\right)$, simply related to the electron (hole) ionization threshold energy $E_{\text {the }}\left(E_{\text {thh }}\right)$ and the electric field, $F$ as

$E_{\text {the }}=\frac{d_{e}}{F}(3)$

(assuming a uniform field), and on the ionization coefficients of carriers after traveling the dead-space. The latter is also referred to as the enabled ionization coefficients, $\alpha^{*}\left(\beta^{*}\right)$. [Estimates for the

quantity $E_{\text {the }}\left(E_{\text {thh }}\right)$ can be obtained from the literature or from the band structure; these can be used in the DSMT as a starting point.] However, despite the availability of $\alpha^{\prime}\left(\beta^{\prime}\right)$ for many semiconductors, these coefficients are not applicable to the DSMT model, as there is no simple relationship relating the experimentally determined $\alpha^{\prime}\left(\beta^{\prime}\right)$ and $\alpha^{*}\left(\beta^{*}\right)$. Historically, obtaining $\alpha^{*}\left(\beta^{*}\right)$ and $E_{\text {the }}\left(E_{\text {thh }}\right)$ has required the experimental values of $\left.M_{e}\left(M_{h}\right)\right)$ and $F_{e}\left(F_{h}\right)$ in a series of $\mathrm{p}$-i-n devices of different thicknesses and a fitting procedure using the DSMT model [13]. While this procedure works, it requires the accurate generation of considerable new noise data in many materials.

In this paper, we show that $\alpha^{*}\left(\beta^{*}\right)$ can be approximated from experimentally determined $\alpha^{\prime}\left(\beta^{\prime}\right)$ using a simple equation, provided that a reasonably accurate knowledge of $E_{\text {the }}\left(E_{\text {thh }}\right)$ exists without the need for any excessnoise data. The parameter $\alpha^{\prime}\left(\beta^{\prime}\right)$ is determined from multiplication measurements on $p-i-n$ photodiodes with a uniform electric field as they simplify the subsequent discussions. These estimates of $\alpha^{*}\left(\beta^{*}\right)$ can, in turn, be used in the DSMT models to generate the multiplication and excess noise in p-i-n devices as thin as $0.05 \mu \mathrm{m}$. They can also be used to predict the breakdown probability and breakdown voltage [14], [15], as well as the statistical characteristics of the time response of the APD [16], [17].

\section{SECTION II. Model}

In the DSMT model, the electron ionization pdf, $h_{e}(x)$ is described as a perfect exponential function after the carrier traverses the dead-space distance, $d_{e}[11]$

$h_{e}(x)= \begin{cases}0, & x \leq d_{e} \\ \alpha^{*} \exp \left[-\alpha^{*}\left(x-d_{e}\right)\right], & x>d_{e}(4)\end{cases}$

where the corresponding mean ionizing path length, $x_{e}$ is $d_{e}+1 / \alpha^{*}$. However, the mean ionization path length between successive electron ionizations, $x_{\mathrm{se}}$, is different from $x_{e}$, because as each time an electron ionizes, an offspring electron is launched alongside the parent and they are both set forth to initiate the subsequent ionizations independently of each other after they traverse their individual dead-spaces. To calculate $x_{\text {se, }}$, we must first consider the electron survival probability, $S_{e}(x)$, where an electron survives a distance $\mathrm{x}$ without impact ionizing. This quantity is readily derived from (4) as follows: 
$S_{e}(x)=\int_{x}^{\infty} h_{e}(x) d x= \begin{cases}1, & x \leq d_{e} \\ \exp \left[-\alpha^{*}\left(x-d_{e}\right)\right], & x>d_{e}\end{cases}$

Once an electron impact ionizes and, therefore, gives rise to an offspring electron-hole pair, both the parent, which starts afresh, and the offspring electron are then assigned with survival probabilities $S_{e 1}$ and $S_{e 2}$, respectively. The joint survival probability, $S_{T}(x)$, for the parent and offspring electrons, according to which both electrons travel a distance $x$ without impact ionizing is the product of $S_{e 1}(x)$ and $S_{e 2}(x)$, namely

$S_{T}(x)=S_{e 1}(x) S_{e 1}(x)= \begin{cases}1, & x \leq d_{e} \\ \exp \left[-2 \alpha^{*}\left(x-d_{e}\right)\right], & x>d_{e}(6)\end{cases}$

The ionizing pdf for the electron pair, $h_{T}(x)$, can be obtained by differentiating $1-S_{T}(x)$, which gives

$h_{T 1}(z)= \begin{cases}0, & x \leq d_{e} \\ 2 \alpha^{*} \exp \left[-2 \alpha^{*}\left(x-d_{e}\right)\right], & x>d_{e} .\end{cases}$

Interestingly, the mean here, $x_{\mathrm{se}}$, is $\left(1 / 2 \alpha^{*}\right)+d_{e}$. A similar derivation from (4) to $\underline{6}$ can be repeated in the case of the local model framework where $d_{e}=0$ and

$h_{T 2}(z)=2 \alpha^{\prime} \exp \left[-2 \alpha^{\prime}(x)\right], x>0(7 b)$

is obtained with a mean of $x_{s e}=1 /\left(2 \alpha^{\prime}\right)$. The relationship between $\alpha^{*}\left(\beta^{*}\right)$ and $\alpha^{\prime}\left(\beta^{\prime}\right)$ can now be found by equating the mean ionizing lengths from the DSMT and local model when they are compared at the same electric field in identical $p$-i-n structures, as they should yield the same multiplication value. This gives

$\alpha^{\prime}=\frac{1}{\frac{1}{\alpha^{*}}+2 d_{e}} \cdot(8)$

Note that $\alpha^{\prime}\left(\beta^{\prime}\right)$ given by $(8)$ is not the same as $1 / x_{e}$, as it is derived from $1 / x_{\mathrm{se}}$. This accounts for why there is $2 d_{e}$ rather than just $d_{e}$ in the denominator of (8). The rate $\beta^{\prime}$ can be expressed in a similar manner by replacing $h_{e}(x), \alpha^{*}, d_{e}, x_{\mathrm{se}}$, and $S_{e}(x)$ with $h_{h}(x), \beta^{*}, d_{h}, x_{\mathrm{sh}}$, and $S_{h}(x)$, respectively.

An alternative (more complicated and less intuitive) way to arrive at $(8)$ is as follows. Spinelli and Lacaita [18] attempted to solve the DSMT model to extract the multiplication analytically. This technique involved differentiating the DSMT recurrence equations and then further simplifying them using a perturbation method, which is reasonably accurate for small dead-space to device-width ratios, $d / w$. The quantity $M_{e}\left(M_{h}\right)$ obtained is then expressed in terms of $\alpha^{*}\left(\beta^{*}\right)$ in the presence of dead-space as follows [18]:

$$
\begin{aligned}
& M(w)=M_{e}=\frac{\exp \left(\alpha d_{h}\right)}{1-\int_{d_{e}}^{w-d_{h}} \alpha \exp \left[-\int_{x}^{w-d_{h}}(\alpha-\beta) d x^{\prime}\right] d x} \\
& M(0)=M_{h}=\frac{\exp \left(\beta d_{e}\right)}{1-\int_{d_{e}}^{w-d_{h}} \alpha \exp \left[-\int_{x}^{w-d_{h}}(\alpha-\beta) d x^{\prime}\right] d x}
\end{aligned}
$$

where 


$$
\begin{aligned}
& \alpha=\alpha^{*} \frac{1+2 \beta^{*} d_{h}+\beta^{*} d_{e}}{1+2 \alpha^{*} d_{e}+2 \beta^{*} d_{h}+3 \alpha^{*} \beta^{*} d_{e} d_{h}} \\
& \beta=\beta^{*} \frac{1+2 \alpha^{*} d_{e}+\alpha^{*} d_{h}}{1+2 \alpha^{*} d_{e}+2 \beta^{*} d_{h}+3 \alpha^{*} \beta^{*} d_{e} d_{h}} .
\end{aligned}
$$

The local rates $\alpha$ and $\beta$ from $\underline{(9 \mathrm{a})}$ to $\underline{(10 \mathrm{~b})}$ are valid for $d_{e} \leq x \leq w-d_{h}$ as defined in [18]. From (9a), it is possible to equate the multiplication obtained from solving the recurrence equations (after the perturbation approximation) and the multiplication obtained from the local model (where no dead-space is assumed) for a given $w$ in order to relate $\alpha^{*}$ to $\alpha_{\text {device }}$. The parameter $\alpha_{\text {device }}$ is normally extracted from experiments and, therefore, includes the effect of the dead-space. This is the rate used in the local model which results in multiplication that is equivalent to that obtained from the DSMT model. For simplicity, assuming a perfect $\mathrm{p}$-i-n structure where $M_{e}=M_{h}$ and $F_{e}=F_{h}$, i.e., $\alpha=\beta$, (1) reduces to

$$
M(w)=M_{e}=\frac{1}{1-\alpha_{\text {device }} w}
$$

Similarly, assuming $d_{e}=d_{h}$ and $\alpha^{*}=\beta^{*}$, (9a) simplifies to

$$
M(w)=M_{e} \approx \frac{1+\left(d_{e}+\frac{1}{\alpha^{*}}\right)^{-1} d_{e}}{1-\left(d_{e}+\frac{1}{\alpha^{*}}\right)^{-1}\left(w-2 d_{e}\right)}
$$

By equating the gain expressions in $(11)$ and $(12)$, we obtain

$$
\alpha_{\text {device }} \approx \frac{1-\frac{d_{e}}{w}}{\frac{1}{\alpha^{*}}+2 d_{e}}
$$

The denominator of this expression is identical to that in $(8)$, but the effect of the devicedependent $\alpha_{\text {device }}\left(\beta_{\text {device}}\right)$ is accounted for qualitatively by the $d / w$ term in the numerator. Similar derivations were used in another simple extreme case where $\beta^{*}, d_{h}$ in (9a), and $\beta$ in (1) are set to 0 . These simplified multiplication expressions are equated to each other and $\underline{(13)}$ is once again obtained. It is, therefore, concluded that $\underline{(13)}$ is independent of $\beta^{*}$ and $d_{h}$.

This first-order approximation becomes increasingly inaccurate in thin devices with high gains due to the large $d / w$ ratio; the approximation is, therefore, not a good way for determining the multiplication, as pointed out in [18]. However, if the asymptotes of $\alpha_{\text {device }}$ for devices with different widths (at high field) are considered, i.e., when $d / w$ becomes negligible, then $(13)$ approaches $(8)$. This means that we can extract the enabled (nonlocal) ionization coefficient, $\alpha^{*}\left(\beta^{*}\right)$, from the asymptotes of a family of the experimental coefficients, $\alpha_{\text {device }}$. For each device width, the asymptote can be found when the electric field is high, or equivalently when the multiplication is high.

To verify the validity of (8), we have used a simple Monte Carlo (SMC) model to generate the associated multiplication and noise characteristics in an idealized series of $p$-i-n photodiodes. Several authors showed that such a model agrees well with experimental gain and noise for several semiconductor materials even with $w$ thinner than $0.1 \mu \mathrm{m}$ [19]-[20][21]. Using the input parameters in [19], multiplication $\left(M_{e}\right.$ and $\left.\left.M_{h}\right)\right)$ and noise $\left(F_{e}\right.$ and $\left.F_{h}\right)$ of GaAs perfect $\mathrm{p}$-i-n devices with $w$ of $0.05,0.1,0.2,0.5$, and $1 \mu \mathrm{m}$ were simulated using the SMC model. Details of the SMC model have been reported in [19]. These SMC data were compared against the multiplication and noise data obtained from the random-path-length (RPL) model [12], which utilize the randomly generated ionization path lengths according to the ionizing pdf given by (4). The RPL technique 
essentially gives identical results to the DSMT technique [11], as shown in [22]. The results are described in Section III.

\section{SECTION III. Results and Discussion}

The parameters $\alpha_{\text {device }}\left(\beta_{\text {device}}\right)$ were determined from $M_{e}$ and $\left.M_{h}\right)$ simulated from the SMC model using

$\alpha_{\text {device }}=\frac{1}{w} \frac{M_{e}-1}{M_{e}-M_{h}} \ln \left(\frac{M_{e}}{M_{h}}\right)(14 a)$

and

$\beta_{\text {device }}=\frac{1}{w} \frac{M_{h}-1}{M_{e}-M_{h}} \ln \left(\frac{M_{e}}{M_{h}}\right)$.

The local ionization coefficient $\alpha^{\prime}\left(\beta^{\prime}\right)$ was parameterized using the highest value of $\alpha_{\text {device }}\left(\beta_{\text {device }}\right)$ at a given electric field, which was extracted from multiplication of different thicknesses $p-i-n$ photodiodes, as shown in Fig. 1, to give the device-independent ionization coefficient. The results are tabulated in Table I. These values are similar compared with those in [23], but they cover a wider electric field range. For clarity, $\alpha_{\text {device }}\left(\beta_{\text {device }}\right)$ for only 0.05-, 0.1-, and 1- $\mu \mathrm{m}$ p-i-n photodiodes are shown.
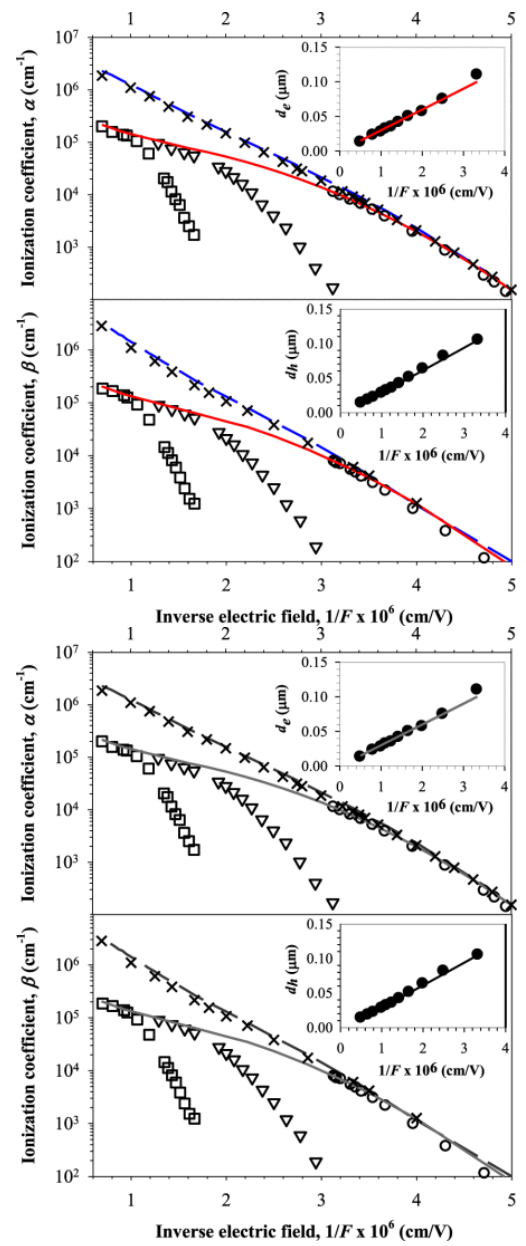

Fig. 1. $\alpha_{\text {device }}\left(\beta_{\text {device}}\right)$ of $0.05,0.1$, and $1 \mu \mathrm{m} \mathrm{p-i-n} \mathrm{photodiodes} \mathrm{calculated} \mathrm{from}(14 \mathrm{a})$ and $(14 \mathrm{~b})$ denoted as $\square, \nabla$, and $\bigcirc$, respectively. Parameterized $\alpha^{\prime}\left(\beta^{\prime}\right), \alpha^{*}\left(\beta^{*}\right)$ determined from $\underline{(8)}$ and $\underline{(15)}$ are shown as solid, medium-dashed lines, and $\times$, respectively. Inset: fittings (lines) of carriers dead-space using (3) with those obtained from the SMC simulations (circles). 
TABLE I $\alpha^{\prime}\left(\beta^{\prime}\right)$ of Group IV and III-V Semiconductors Expressed as $\alpha^{\prime}\left(\beta^{\prime}\right)=A \exp [-(B / F) C]$ Where $F$ is Electric Field. The Ionization Coefficients That Were Found to Have a Wider Electric Field Range Than the Previous Reports are Marked as*. The Threshold Energies Shown are for Secondary lonizing Carriers 


\begin{tabular}{|c|c|c|c|c|c|c|c|}
\hline Material & Electric field range $(\mathrm{kV} / \mathrm{cm})$ & Coefficient Type & $A\left(\times 10^{5} \mathrm{~cm}^{-1}\right)$ & $B\left(\times 10^{5} \mathrm{~cm}^{-1}\right)$ & $C\left(\times 10^{5} \mathrm{~cm}^{-1}\right)$ & $E_{\text {the }}(\mathrm{eV})$ & $E_{\text {the }}(\mathrm{eV})$ \\
\hline \multirow[t]{4}{*}{ Si [36] } & $175-40$ & $\alpha$ & 7.03 & 12.3 & 1.00 & $1.8[26]$ & $2.4[26]$ \\
\hline & & 8 & 15.8 & 20.4 & 1.00 & & \\
\hline & $400-800 *$ & $\alpha$ & 7.03 & 12.3 & 1.00 & & \\
\hline & & 8 & 6.71 & 16.9 & 1.00 & & \\
\hline \multirow[t]{2}{*}{ GaAs [23] } & $150-900$ & $\alpha$ & 2.28 & 677 & 1.51 & $3.0[23]$ & 3.3 \\
\hline & & $B$ & 2.24 & 715 & 1.55 & & \\
\hline \multirow[t]{5}{*}{ GaAs (this work) } & $150-500$ & $\alpha$ & 1.45 & 5.00 & 2.10 & 3.0 & 3.3 \\
\hline & $500-1110$ & $\alpha$ & 4.70 & 12.0 & 0.90 & & \\
\hline & & 8 & 4.00 & 11.0 & 1.00 & & \\
\hline & $1110-1400$ & $\alpha$ & 6.39 & 16.0 & 0.90 & & \\
\hline & & 8 & 5.92 & 15.5 & 0.95 & & \\
\hline \multirow[t]{5}{*}{$\ln P[37]$} & $240-380$ & $\alpha$ & 112 & 31.1 & 1.00 & $2.8[27]$ & $3.0[27]$ \\
\hline & & 8 & 47.9 & 25.5 & 1.00 & & \\
\hline & $380-560$ & $\alpha$ & 29.3 & 26.4 & 1.00 & & \\
\hline & $560-1250 *$ & $\alpha$ & 2.32 & 8.46 & 2.00 & & \\
\hline & & 8 & 2.48 & 7.89 & 2.00 & & \\
\hline \multirow[t]{2}{*}{$\mathrm{SiC}$} & $1000-4000$ & $\alpha$ & 7.00 & 70.0 & 1.66 & $12.0[28]$ & $8.0[28]$ \\
\hline & & $B$ & 19.0 & 103 & 1.01 & & \\
\hline \multirow[t]{2}{*}{$\mathrm{Al}_{0.6} \mathrm{Ga}_{0.4 \mathrm{As}}[23]$} & $330-1100$ & $\alpha$ & 2.95 & 11.6 & 1.44 & $3.4[29]$ & $3.6[29]$ \\
\hline & & 8 & 3.11 & 12.1 & 1.46 & & \\
\hline \multirow[t]{4}{*}{$\mathrm{Al}_{0.8 \mathrm{Ga}} \mathrm{Ga}_{0.2 \mathrm{~s}}[8]$} & $328-1110$ & $\alpha$ & 3.18 & 10.4 & 1.67 & 3.2 & 2.3 \\
\hline & & 8 & 3.55 & 11.2 & 1.85 & & \\
\hline & $1110-1540$ & $\alpha$ & 38.4 & 102 & 0.55 & & \\
\hline & & 8 & 38.4 & 102 & 0.55 & & \\
\hline \multirow[t]{2}{*}{$\ln _{0.52} \mathrm{Al}_{0.48} \mathrm{As}[38]$} & $220-980$ & $\alpha$ & 2.20 & 8.90 & 1.71 & $3.2[31]$ & $3.5[31]$ \\
\hline & & $B$ & 2.95 & 11.5 & 1.71 & & \\
\hline \multirow[t]{2}{*}{$G_{0.52} \ln _{0.48 \mathrm{P}}[39]$} & $357-1700$ & $\alpha$ & 4.57 & 14.1 & 1.73 & $4.1[32]$ & $4.1[32]$ \\
\hline & & 8 & 4.73 & 14.3 & 1.65 & & \\
\hline Al0.52In $0.48 \mathrm{P}[40]$ & $40-1300 *$ & $\alpha$ & 4.93 & 16.5 & 1.78 & 4.6 & 4.6 \\
\hline
\end{tabular}




\begin{tabular}{|c|c|c|c|c|c|c|c|}
\hline Material & $\begin{array}{l}\text { Electric field range } \\
(\mathrm{kV} / \mathrm{cm})\end{array}$ & $\begin{array}{l}\text { Coefficient } \\
\text { Type }\end{array}$ & $A\left(\times 10^{3} \mathrm{~cm}^{-1}\right)$ & $B\left(\times 10^{5} \mathrm{~cm}^{-1}\right)$ & $C\left(\times 10^{3} \mathrm{~cm}^{-1}\right)$ & $E_{\text {sut }}(\mathrm{eV})$ & $E_{\mathrm{atat}}(\mathrm{eV})$ \\
\hline \multirow[t]{4}{*}{$\mathrm{Si}[36]$} & $175-400$ & $a$ & 7.03 & 12.3 & 1.00 & $1,8[26]$ & $2.4[26]$ \\
\hline & & $\beta$ & 15.8 & 20.4 & 1.00 & & \\
\hline & $400-800^{*}$ & $\alpha$ & 7.03 & 12.3 & 1.00 & & \\
\hline & & $\beta$ & 6.71 & 16.9 & 1.00 & & \\
\hline \multirow[t]{2}{*}{ GaAs [23] } & $150-900$ & $\alpha$ & 2.28 & 677 & 1.51 & $3.0[23]$ & 3.3 \\
\hline & & $\beta$ & 2.24 & 715 & 1.55 & & \\
\hline \multirow{6}{*}{$\begin{array}{l}\text { GaAs (this } \\
\text { work) }\end{array}$} & $150-500$ & $a$ & 1.45 & 5.00 & 2.10 & 3.0 & 3.3 \\
\hline & & $\beta$ & 1.55 & 5.50 & 2.00 & & \\
\hline & $500-1110$ & $\alpha$ & 4.70 & 12.0 & 0.90 & & \\
\hline & & $\beta$ & 4.00 & 11.0 & 1.00 & & \\
\hline & $1110-1400$ & $a$ & 6.39 & 16.0 & 0.90 & & \\
\hline & & $\beta$ & 5.92 & 15.5 & 0.95 & & \\
\hline \multirow[t]{6}{*}{$\ln P[37]$} & $240-380$ & $\alpha$ & 112 & 31.1 & 1.00 & $2.8[27]$ & $3.0[27]$ \\
\hline & & $\beta$ & 47.9 & 25.5 & 1.00 & & \\
\hline & $380-560$ & $\alpha$ & 29.3 & 26.4 & 1.00 & & \\
\hline & & $\beta$ & 16.2 & 21.1 & 1.00 & & \\
\hline & $560-1250^{\circ}$ & $\alpha$ & 2.32 & 8.46 & 2.00 & & \\
\hline & & $\beta$ & 2.48 & 7.89 & 2.00 & & \\
\hline \multirow[t]{2}{*}{$\mathrm{SiC}$} & $1000-4000$ & $\alpha$ & 7.00 & 70.0 & 1.66 & $12.0[28]$ & $8.0[28]$ \\
\hline & & $\beta$ & 19.0 & 103 & 1.01 & & \\
\hline \multirow{2}{*}{$\begin{array}{c}\mathrm{Al}_{06} \mathrm{Ga}_{0,4} \mathrm{As} \\
{[23]}\end{array}$} & $330-1100$ & $\alpha$ & 2.95 & 11.6 & 1.44 & $3.4[29]$ & $3.6[29]$ \\
\hline & & $\beta$ & 3.11 & 12.1 & 1.43 & & \\
\hline \multirow{4}{*}{$\begin{array}{c}\mathrm{Al}_{68} \mathrm{Ga}_{62} \mathrm{As} \\
{[8]}\end{array}$} & $328-1110$ & $\alpha$ & 3.18 & 10.4 & 1.67 & 3.2 & 2.3 \\
\hline & & $\beta$ & 3.55 & 11.2 & 1.85 & & \\
\hline & $1110-1540$ & $\alpha$ & 38.4 & 102 & 0.55 & & \\
\hline & & $\beta$ & 38.4 & 102 & 0.55 & & \\
\hline \multirow{2}{*}{$\begin{array}{c}\mathrm{In}_{6.52} \mathrm{Al}_{0.48} \mathrm{As} \\
{[38]} \\
\end{array}$} & $220-980$ & $\alpha$ & 2.20 & 8.90 & 1.71 & $3.2[31]$ & $3.5[3 \mathrm{II}]$ \\
\hline & & $\beta$ & 2.95 & 11.5 & 1.71 & & \\
\hline \multirow{2}{*}{$\begin{array}{c}\mathrm{Ga}_{0.52} \ln _{0.48} \mathrm{P} \\
{[39]} \\
\end{array}$} & $357-1700$ & $\alpha$ & 4.57 & 14.1 & 1.73 & $4.1[32]$ & $4.1[32]$ \\
\hline & & $\beta$ & 4.73 & 14.3 & 1.65 & & \\
\hline \multirow{2}{*}{$\begin{array}{c}\mathrm{Al}_{0, s_{2}} \ln _{6,43} \mathrm{P} \\
{[40]}\end{array}$} & $400-1300^{*}$ & $\alpha$ & 4.93 & 16.5 & 1.78 & 4.6 & 4.6 \\
\hline & & $\beta$ & 5.29 & 15.9 & 1.98 & & \\
\hline
\end{tabular}


The SMC model can also generate the ionizing pdf at a given electric field by logging the ionizing length between two successive ionizing events, i.e., xe , as shown in Fig. 2, which gives the mean electron ionizing length, $1 / \alpha_{\mathrm{MC}}$. This can be done for primary ionizing carriers, injected with just thermal energy and for those secondary ionizing carriers which can start with significant residual energy [24].
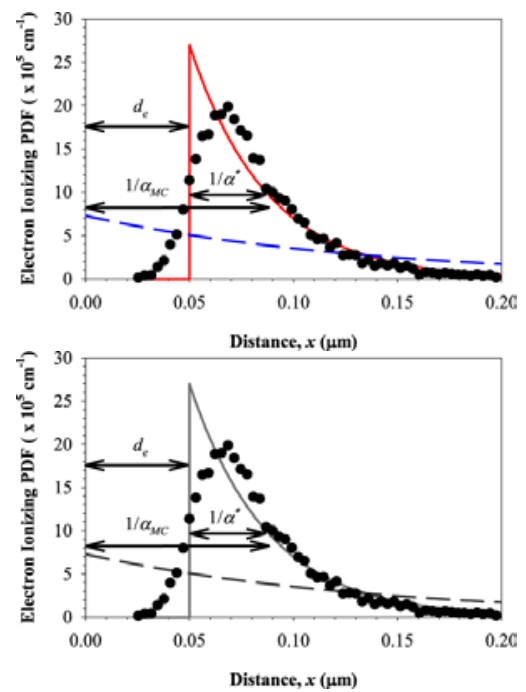

Fig. 2. Electron ionizing pdf of GaAs at $600 \mathrm{kV} / \mathrm{cm}$ obtained from the $\mathrm{SMC}$ simulations $(\bullet)$. Solid line: mean ionizing length $\left(x_{e}=1 / \alpha_{\mathrm{MC}}\right)$ and dead-space $\left(d_{e}\right)$ were determined to calculate $\alpha^{*}$ using $(15)$ to generate the pdf using (4). Dashed lines: $\alpha^{\prime}$ obtained using (8).

The pdf at the same electric field $(600 \mathrm{kV} / \mathrm{cm})$ is approximated in the DSMT model in Fig. 2 using (4), which comprises $d_{e}$ followed by an exponential function that has a mean of $1 / \alpha^{*}$. The dead-space $d_{e}$ was defined as the distance where the rising edge of the pdf reached $50 \%$ of its peak value [25]; therefore, $E_{\text {the }}\left(E_{\text {thh }}\right)$ for secondary ionizing carriers was calculated as 3 (3.3) eV using (3), as shown in the inset of Fig. 1, while the initial carrier threshold energies, $E_{\text {the }}\left(E_{\text {thh }}\right)$ determined from the SMC model was $\sim 15 \%$ higher than those for the secondary carriers at 3.5 (3.8) eV (using the secondary carrier threshold energy for the primary injected carrier will result in a slight overestimation of the low multiplication values in devices but it will not change the breakdown voltage appreciably). The enabled ionization coefficient $\alpha^{*}\left(\beta^{*}\right)$ can, then, be determined from $\alpha_{\mathrm{MC}}\left(\beta_{\mathrm{MC}}\right)$ using

$\frac{1}{\alpha_{\mathrm{MC}}}=d_{e}+\frac{1}{\alpha^{*}}(15)$

and this is plotted as a function of electric field for secondary ionizing carriers in Fig. 1.

The ionizing pdf associated with $\alpha^{\prime}$ is also shown in Fig. 2, where the peak is significantly lower than those from the SMC and DSMT models as this assumes that secondary carriers have no dead-space and, therefore, are allowed to ionize immediately after they are created.

Using (8) with $E_{\text {the }}\left(E_{\text {thh }}\right)$ of $3(3.3) \mathrm{eV}$ on $\alpha^{\prime}\left(\beta^{\prime}\right)$ shows that excellent agreement to the $\alpha^{*}\left(\beta^{*}\right)$, which were determined from the SMC simulations, can be achieved over a wide range of electric fields from $200 \mathrm{kV} / \mathrm{cm}$ to 1.4 MV/cm, as shown in Fig. 1. We reiterate that these values can be used as input parameters to the DSMT model to generate multiplication and excess noise. Using the appropriate $E_{\text {the }}\left(E_{\text {thh }}\right)$ for primary and secondary carriers, the calculated DSMT-based multiplication characteristics agree well with those obtained from the SMC simulations even for a $0.05-\mu \mathrm{m}$ thick p-i-n over several orders of magnitude, as shown in Fig. 3. 

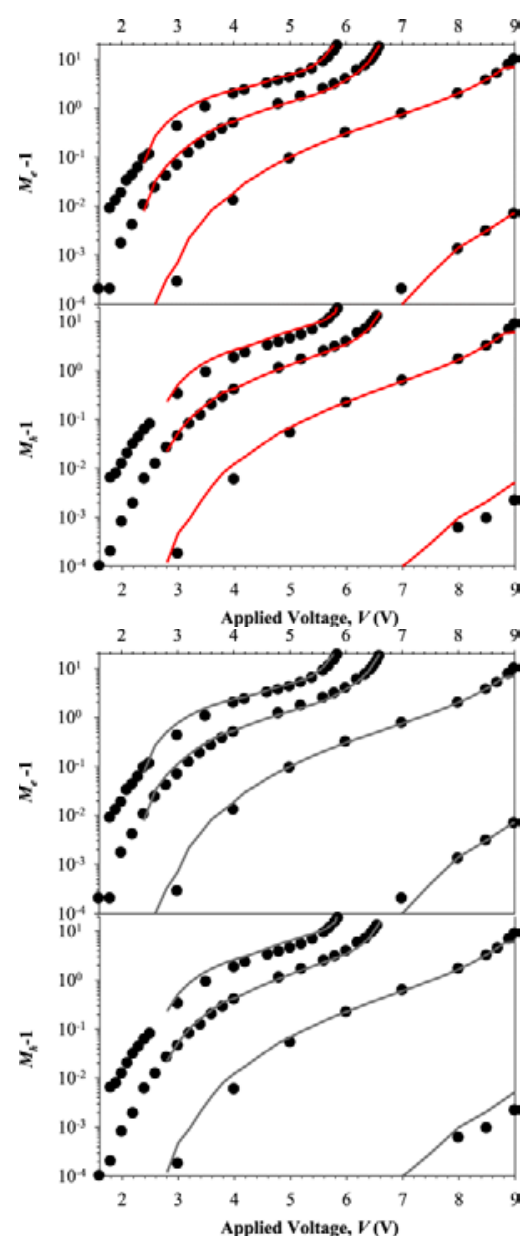

Fig. 3. $M_{e}-1$ and $M_{h}-1$ curves of $p$-i-n photodiodes with w of $0.05,0.1,0.2$, and $0.5 \mu \mathrm{m}$ (from left to right) simulated by the RPL (solid lines) and SMC model $(\bullet)$. To remain clarity, the multiplication of $1-\mu \mathrm{m} p-i-n$ is not shown.

Furthermore, excess noise factors calculated from the DSMT recurrence model showed good agreement with those obtained from the SMC simulations down to 0.1- $\mu \mathrm{m}$ p-i-n, as shown in Fig. 4. As the device width shrinks further to $0.05 \mu \mathrm{m}$, the DSMT model underestimates both $F_{e}\left(F_{h}\right)$ and shows no multiplication at low electric fields because of the hard dead-space assumption. In reality, the ionizing pdf is not accurately presented by (4) even at a relatively low field of $600 \mathrm{kV} / \mathrm{cm}$ as shown in Fig. 2, where it initially rises to the peak value gradually due to the soft dead-space and thereafter decays exponentially. 

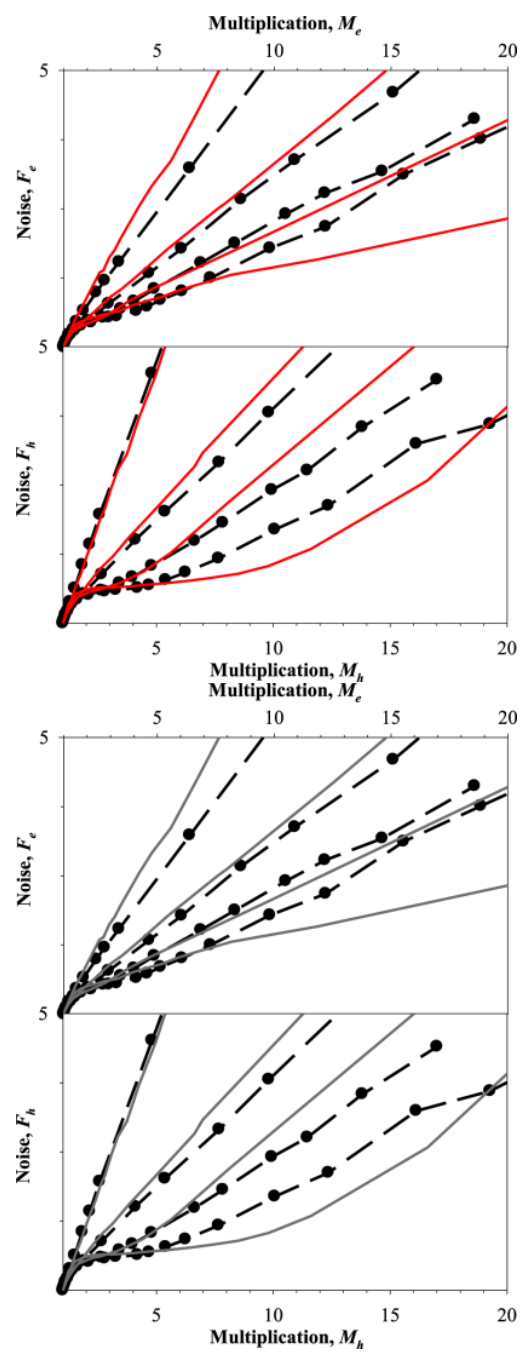

Fig. 4. Excess noise of $p$-i-n photodiodes with w of $0.05,0.1,0.2$, and $1 \mu \mathrm{m}$ (from top to bottom) due to pure electron and hole injection, $F_{e}$ and $F_{h}$, respectively, simulated by the RPL (solid lines) and SMC model (symbols with dashed lines).

Considering the simplicity of $\left(\underline{8)}\right.$ in estimating $\alpha^{*}\left(\beta^{*}\right)$, simulated results of multiplication and excess noise factor from the DSMT model for a wide range of semiconductor materials like Si [26], InP [27], SiC [28], $\mathrm{Al}_{0.6} \mathrm{Ga}_{0.4} \mathrm{As}$ [29], $\mathrm{Al}_{0.8} \mathrm{Ga}_{0.4} \mathrm{As}$ [30], $\ln _{0.52} \mathrm{Al}_{0.48} \mathrm{As}$ [31], and $\mathrm{Ga}_{0.52} \ln _{0.48} \mathrm{P}$ [32] agree surprisingly well with the experimental data, provided good knowledge of $\alpha^{\prime}\left(\beta^{\prime}\right)$ and their corresponding $E_{\text {the }}\left(E_{\text {thh }}\right)$ exists, as shown in Table I. The effective threshold energy in the DSMT model is the mean energy carriers attain before impact ionization, and as such it differs from other definitions in [33]-[34][35]. The threshold energy $E_{\text {the }}\left(E_{\text {thh }}\right)$ can be obtained either from excess-noise measurements or from knowledge of the multiplication over a wide dynamic range, when the effects of the dead-space become important.

\section{SECTION IV. Conclusion}

Given the ionization threshold energies, the enabled $\alpha^{*}\left(\beta^{*}\right)$ required by the DSMT model can be recovered from experimentally determined $\alpha^{\prime}\left(\beta^{\prime}\right)$ using the simple relationship in (8), which can then be used in the DSMT model to predict the mean multiplication, excess noise factor, the breakdown probability, and the breakdown voltage. With the consideration of slightly different threshold energies for primary and secondary carriers, multiplication and noise data calculated from the DSMT model fit well with the SMC results, even for a $0.05-\mu m$ thick p-i-n and while assuming a hard dead-space ionization pdf. The proposed technique for extracting the 
enabled ionization coefficients relies only on the knowledge of multiplication data and does not require explicit knowledge of the excess-noise characteristics.

\section{ACKNOWLEDGMENT}

The authors would like to thank Prof. G. J. Rees for many useful discussions.

\section{References}

1. R. J. McIntyre, "Multiplication noise in uniform avalanche diodes", IEEE Trans. Electron Devices, vol. ED-13, no. 1, pp. 164-168, Jan. 1966, [online] Available: http://ieeexplore.ieee.org/stamp/stamp.jsp?arnumber=1474241.

2. G. E. Stillman, C. M. Wolfe, R. K. Willardson, A. C. Beer, "Avalanche photodiodes" in Semiconductors and Semimetals, New York, NY, USA:Academic, vol. 12, pp. 333, 1977.

3. M. H. Woods, W. C. Johnson, M. A. Lampert, "Use of a Schottky barrier to measure impact ionization coefficients in semiconductors", Solid-State Electron., vol. 16, no. 3, pp. 381-394, Mar. 1973, [online] Available: http://www.sciencedirect.com/science/article/pii/0038110173900130\#.

4. W. N. Grant, "Electron and hole ionization rates in epitaxial silicon at high electric fields", Solid-State Electron., vol. 16, no. 10, pp. 1189-1203, Oct. 1973, [online] Available: http://www.sciencedirect.com/science/article/pii/0038110173901470.

5. G. E. Bulman, V. M. Robbins, G. E. Stillman, "The determination of impact ionization coefficients in (100) gallium arsenide using avalanche noise and photocurrent multiplication measurement", IEEE Trans. Electron Devices, vol. 32, no. 11, pp. 2454-2466, Nov. 1985, [online] Available: http://ieeexplore.ieee.org/stamp/stamp.jsp?arnumber=1485041.

6. Y. Okuto, C. R. Crowell, "Ionization coefficients in semiconductors: A nonlocalized property", Phys. Rev. B, vol. 10, no. 10, pp. 4284-4296, Nov. 1974, [online] Available: http://journals.aps.org/prb/pdf/10.1103/PhysRevB.10.4284.

7. R. M. Flitcroft, J. P. R. David, P. A. Houston, C. C. Button, "Avalanche multiplication in GalnP/GaAs single heterojunction bipolar transistors", IEEE Trans. Electron Devices, vol. 45, no. 6, pp. 1207-1212, Jun. 1998, [online] Available: http://ieeexplore.ieee.org/stamp/stamp.jsp?tp=\&arnumber=678515.

8. B. K. Ng et al., " Avalanche multiplication characteristics of Al $0.8 \mathrm{Ga} 0.2$ As diodes ", IEEE Trans. Electron Devices, vol. 48, no. 10, pp. 2198-2204, Oct. 2001, [online] Available: http://ieeexplore.ieee.org/stamp/stamp.jsp?arnumber=954454.

9. C. Hu, K. A. Anselm, B. G. Streetman, J. C. Campbell, "Noise characteristics of thin multiplication region GaAs avalanche photodiodes", Appl. Phys. Lett., vol. 69, no. 24, pp. 3734-3736, Dec. 1996, [online] Available: http://scitation.aip.org/content/aip/journal/apl/69/24/10.1063/1.117205.

10. K. F. Li et al., " Avalanche multiplication noise characteristics in thin GaAs $\mathrm{p}+-\mathrm{i}-\mathrm{n}+$ diodes ", IEEE Trans. Electron Devices, vol. 45, no. 10, pp. 2102-2107, Oct. 1998, [online] Available: http://ieeexplore.ieee.org/stamp/stamp.jsp?tp=\&arnumber $=725242$.

11. M. M. Hayat, B. E. A. Saleh, M. C. Teich, "Effect of dead space on gain and noise of double-carriermultiplication avalanche photodiodes", IEEE Trans. Electron Devices, vol. 39, no. 3, pp. 546-552, Mar. 1992, [online] Available: http://ieeexplore.ieee.org/stamp/stamp.jsp?arnumber=123476.

12. D. S. Ong, K. F. Li, G. J. Rees, J. P. R. David, P. N. Robson, "A simple model to determine multiplication and noise in avalanche photodiodes", J. Appl. Phys., vol. 83, no. 6, pp. 3426-3428, Mar. 1998, [online] Available: http://scitation.aip.org/content/aip/journal/jap/83/6/10.1063/1.367111.

13. M. A. Saleh et al., "Impact-ionization and noise characteristics of thin III-V avalanche photodiodes", IEEE Trans. Electron Devices, vol. 48, no. 12, pp. 2722-2731, Dec. 2001, [online] Available: http://ieeexplore.ieee.org/stamp/stamp.jsp?tp=\&arnumber=974696.

14. M. M. Hayat et al., "Breakdown probabilities for thin heterostructure avalanche photodiodes", IEEE J. Quantum Electron., vol. 39, no. 1, pp. 179-185, Jan. 2003, [online] Available: http://ieeexplore.ieee.org/stamp/stamp.jsp?tp=\&arnumber=1158822. 
15. M. A. Saleh et al., "Breakdown voltage in thin III-V avalanche photodiodes", Appl. Phys. Lett., vol. 79, no. 24, pp. 4037-4039, Dec. 2001, [online] Available:

http://scitation.aip.org/content/aip/journal/apl/79/24/10.1063/1.1425463.

16. M. M. Hayat et al., "Gain-bandwidth characteristics of thin avalanche photodiodes", IEEE Trans. Electron Devices, vol. 49, no. 5, pp. 4037-4039, May 2002, [online] Available: http://ieeexplore.ieee.org/stamp/stamp.jsp?arnumber=998583.

17. C. Groves, C. H. Tan, J. P. R. David, G. J. Rees, M. M. Hayat, "Exponential time response in analogue and Geiger mode avalanche photodiodes", IEEE Trans. Electron Devices, vol. 52, no. 7, pp. 1527-1534, Jul. 2005, [online] Available: http://ieeexplore.ieee.org/stamp/stamp.jsp?tp=\&arnumber=1459115.

18. A. Spinelli, A. L. Lacaita, "Mean gain of avalanche photodiodes in a dead space model", IEEE Trans. Electron Devices, vol. 43, no. 1, pp. 23-30, Jan. 1996, [online] Available: http://ieeexplore.ieee.org/stamp/stamp.jsp?arnumber $=477589$.

19. S. A. Plimmer, J. P. R. David, D. S. Ong, K. F. Li, "A simple model for avalanche multiplication including deadspace effects", IEEE Trans. Electron Devices, vol. 46, no. 4, pp. 769-775, Apr. 1999, [online] Available: http://ieeexplore.ieee.org/stamp/stamp.jsp?arnumber=753712.

20. X. Zhou, J. S. Ng, C. H. Tan, "A simple Monte Carlo model for prediction of avalanche multiplication process in silicon", J. Instrum., vol. 7, no. 8, pp. 1-10, Aug. 2012, [online] Available: http://iopscience.iop.org/17480221/7/08/P08006/pdf/1748-0221_7_08_P08006.pdf.

21. S. C. Liew Tat Mun, C. H. Tan, Y. L. Goh, A. R. J. Marshall, J. P. R. David, "Modeling of avalanche multiplication and excess noise factor in In0.52Al0.48As avalanche photodiodes using a simple Monte Carlo model", J. Appl. Phys., vol. 104, no. 1, pp. 013114-1-013114-6, Jul. 2008, [online] Available: http://scitation.aip.org/content/aip/journal/jap/104/1/10.1063/1.2952003.

22. J. S. Ng et al., "Effect of dead space on avalanche speed [APDs]", IEEE Trans. Electron Devices, vol. 49, no. 4, pp. 544-549, Apr. 2002, [online] Available: http://ieeexplore.ieee.org/stamp/stamp.jsp?arnumber=992860.

23. S. A. Plimmer, J. P. R. David, G. J. Rees, P. N. Robson, " Ionization coefficients in Al x Ga 1-x As ( $x=0-0.60)$ ", Semicond. Sci. Technol., vol. 15, no. 7, pp. 692-699, Jul. 2000, [online] Available: http://iopscience.iop.org/0268-1242/15/7/307/pdf/0268-1242_15_7_307.pdf.

24. D. S. Ong, K. F. Li, S. A. Plimmer, G. J. Rees, J. P. R. David, P. N. Robson, " Full band Monte Carlo modeling of impact ionization avalanche multiplication and noise in submicron GaAs $\mathrm{p}+-\mathrm{i}-\mathrm{n}+$ diodes ", J. Appl. Phys., vol. 87, no. 11, pp. 7885-7891, Jun. 2000, [online] Available:

http://scitation.aip.org/content/aip/journal/jap/87/11/10.1063/1.373472.

25. A. Spinelli, A. Pacelli, A. L. Lacaita, "Dead space approximation for impact ionization in silicon", Appl. Phys. Lett., vol. 69, no. 24, pp. 3707-3709, Dec. 1996, [online] Available: http://scitation.aip.org/content/aip/journal/apl/69/24/10.1063/1.117196.

26. C. H. Tan et al., " Avalanche noise measurement in thin Si p +-i-n + diodes ", Appl. Phys. Lett., vol. 76, no. 26, pp. 3926-3928, Jun. 2000, [online] Available: http://scitation.aip.org/content/aip/journal/apl/76/26/10.1063/1.126823.

27. L. J. J. Tan, J. S. Ng, C. H. Tan, J. P. R. David, "Avalanche noise characteristics in submicron InP diodes", IEEE J. Quantum Electron., vol. 44, no. 4, pp. 378-382, Apr. 2008, [online] Available: http://ieeexplore.ieee.org/stamp/stamp.jsp?arnumber=4456802.

28. B. K. Ng et al., "Nonlocal effects in thin $4 \mathrm{H}-\mathrm{SiC}$ UV avalanche photodiodes", IEEE Trans. Electron Devices, vol. 50, no. 8, pp. 1724-1732, Aug. 2003, [online] Available: http://ieeexplore.ieee.org/stamp/stamp.jsp?arnumber=1218663.

29. C. H. Tan, J. P. R. David, S. A. Plimmer, G. J. Rees, R. C. Tozer, R. Grey, "Low multiplication noise thin Al0.6Ga0.4As avalanche photodiodes", IEEE Trans. Electron Devices, vol. 48, no. 7, pp. 1310-1317, Jul. 2001, [online] Available: http://ieeexplore.ieee.org/stamp/stamp.jsp?tp=\&arnumber=930644.

30. B. K. Ng, J. P. R. David, R. C. Tozer, M. Hopkinson, G. Hill, G. J. Rees, " Excess noise characteristics of Al $0.8 \mathrm{Ga}$ 0.2 As avalanche photodiodes ", IEEE Photon. Technol. Lett., vol. 14, no. 4, pp. 522-524, Apr. 2002, [online] Available: http://ieeexplore.ieee.org/stamp/stamp.jsp?tp=\&arnumber=992598. 
31. Y. L. Goh et al., " Excess avalanche noise in In 0.52 A 10.48 As ", IEEE J. Quantum Electron., vol. 43, no. 6, pp. 503-507, Jun. 2007, [online] Available: http://ieeexplore.ieee.org/stamp/stamp.jsp?tp=\&arnumber $=4207490$.

32. C. H. Tan, R. Ghin, J. P. R. David, G. J. Rees, M. Hopkinson, " The effect of dead space on gain and excess noise in In 0.48 Ga $0.52 \mathrm{P} \mathrm{p}+$ in + diodes ", Semicond. Sci. Technol., vol. 18, no. 8, pp. 803-806, Jul. 2003, [online] Available: http://iopscience.iop.org/0268-1242/18/8/314/pdf/0268-1242_18_8_314.pdf.

33. F. Capasso, R. K. Willardson, A. C. Beer, "Physics of avalanche photodiodes" in Semiconductors and Semimetals, New York, NY, USA:Academic, vol. 22, pp. 1-15, 1985.

34. J. R. Hauser, "Threshold energy for avalanche multiplication in semiconductors", J. Appl. Phys., vol. 37, no. 2, pp. 507-509, Feb. 1966, [online] Available: http://scitation.aip.org/content/aip/journal/jap/37/2/10.1063/1.1708204.

35. C. L. Anderson, C. R. Crowell, "Threshold energies for electronhole pair production by impact ionization in semiconductors", Phys. Rev. B, vol. 5, no. 6, pp. 2267-2272, Mar. 1972, [online] Available: http://journals.aps.org/prb/pdf/10.1103/PhysRevB.5.2267.

36. R. Van Overstraeten, H. D. Man, " Measurement of the ionization rates in diffused silicon p-n junctions ", Solid-State Electron., vol. 13, no. 5, pp. 583-608, May 1970, [online] Available: http://www.sciencedirect.com/science/article/pii/0038110170901395\#.

37. L. W. Cook, G. E. Bulman, G. E. Stillman, "Electron and hole impact ionization coefficients in InP determined by photomultiplication measurements", Appl. Phys. Lett., vol. 40, no. 7, pp. 589-591, Jan. 1982, [online] Available: http://scitation.aip.org/content/aip/journal/apl/40/7/10.1063/1.93190.

38. Y. L. Goh et al., "Avalanche multiplication in InAIAs", IEEE Trans. Electron Devices, vol. 54, no. 1, pp. 11-16, Jan. 2007, [online] Available: http://ieeexplore.ieee.org/stamp/stamp.jsp?tp=\&arnumber=4039695.

39. R. Ghin et al., " Avalanche multiplication and breakdown in Ga 0.52 In 0.48 P diodes ", IEEE Trans. Electron Devices, vol. 45, no. 10, pp. 2096-2101, Oct. 1998, [online] Available: http://ieeexplore.iee.org/stamp/stamp.jsp?tp=\&arnumber=725241.

40. J. S. L. Ong, J. S. Ng, A. B. Krysa, J. P. R. David, " Impact ionization coefficients of Al 0.52 In 0.48 P ", IEEE Electron Device Lett., vol. 32, no. 11, pp. 1528-1530, Nov. 2011, [online] Available: http://ieeexplore.ieee.org/stamp/stamp.jsp?arnumber=6017097. 